\title{
Case Study on Type A Niemann Pick Disease
}

\author{
${ }^{2}$ S.Sriram, ${ }^{1}$ Jaleel Ahmed, ${ }^{3}$ Saranya Saminathan,${ }^{3}$ Sweatha Annie, ${ }^{3}$ Silpa Raj \\ ${ }^{I}$ Consultant Paediatrician, Sri Ramakrishna Hospital, Coimbatore. \\ ${ }^{2}$ Department of Pharmacy Practice, SRIPMS, Coimbatore \\ ${ }^{3}$ Pharm D Interns, College of Pharmacy, SRIPMS, Coimbatore
}

\begin{abstract}
Niemann pick disease is a congenital disorder which occurs due to defects in the lysosomal neurovisceral storage characterised by a deficiency in the enzyme, acid sphignomyelinase. This further results in the accumulation of sphignomyelin thus leading to organ failure due to progressive cellular apoptosis. There are mainly three types of NPD namely type-A,B, $C 1$, and $C 2$ out of which $A$ is very rare and no treatment has been so far available which makes it a fatal disease of infancy. This case report encompasses a 30 month old female patient, who presented with intermittent fever, bilateral swelling of the feet, abdominal distress, prominent periorbital swelling and other symptoms that define type-A NPD. The onset of NPD type-A occurs at a very young age with symptoms commencing as early as 3 months and eventually death within 3 years of age. Although the patient's life may not be saved, early diagnosis and management of complications can significantly improve the life expectancy.
\end{abstract}

\section{Introduction}

Niemann pick disease is a congenital disorder characterized by defects in the lysosomal neurovisceral storage. It can be described as a lipid storage disorder, which attributes to the deficiency of a lysosomal enzyme, acid sphingomyelinase. On the contrary, a subtype of it is manifested by the inability to metabolize the cellular cholesterol and other lipids. Apart from this, an abnormality in the intracellular transport of endocytosed cholesterol adds on contributing it to multiple organ failure as a consequence. ${ }^{[1]}$

\section{Significance of Acid Sphingomyelinase}
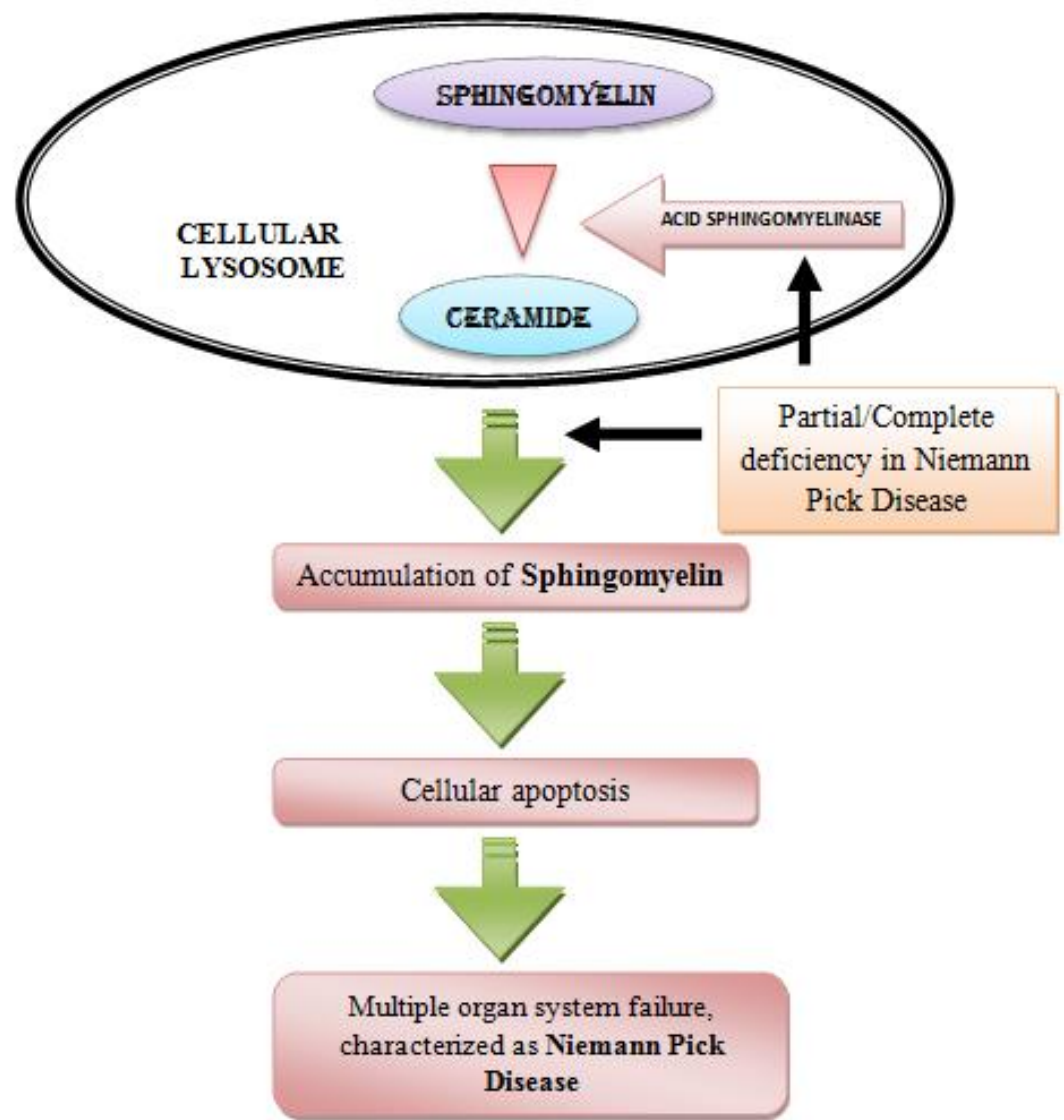
Genetic involvement and inheritance:

Niemann Pick Disease is classified into 3 Major categories.

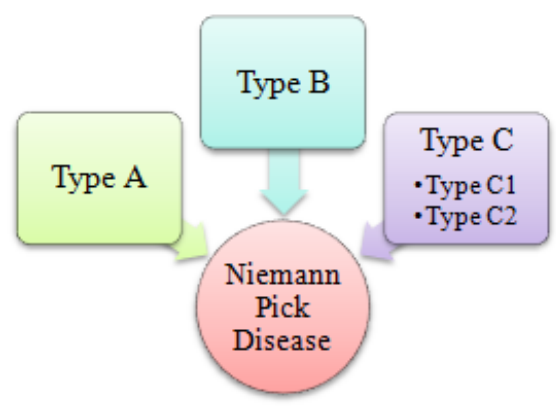

Figure 1- Types of Niemann Pick Disease

This difference in the hierarchy can be defined with respect to their diversification in the responsible genes and in a way through their onset and severity. ${ }^{[1,3]}$

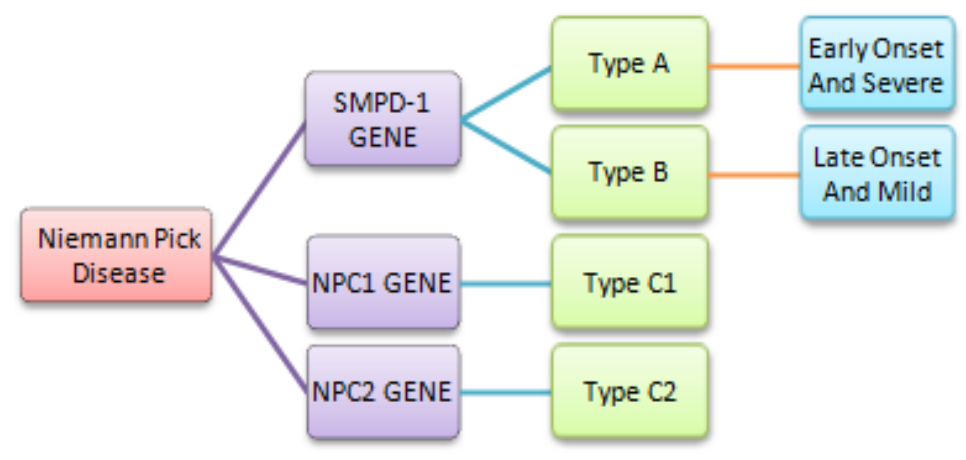

Figure 2- NPD and their Genetic causes

The inheritance of NPD is similar to an autosomal recessive pattern. This indicates both copies of the gene of the patient have mutations, i.e. the parents of the person with NPD carry one copy of the mutated gene, but they typically fail to show signs and symptoms of the condition. ${ }^{[3-4]}$

Presenting Case: A 30- month old female patient was presented to the paediatric OPD with complaints of intermittent fever, bilateral swelling of the feet and characteristic abdominal distress. The symptoms were evident since quite a few months. The history interview helped us to know the child is the $1^{\text {st }}$ kid of a $3^{\text {rd }}$ degree consanguineous marriage. The patient's birth history was uneventful. She was found to be an active, healthy infant until $4^{\text {th }}$ month and was immunized for age. It's after this, that she started to repeal social gatherings and a complete abstinence to smile was observed.

Clinical interpretation: On evaluation in the hospital, she was found to have a progressive hypotension and deteriorating neurological status. Periorbital swelling was another prominent countenance. No similar history was seen in the past. On physical examination, she was conscious, with noticeable pallor and icteric features. Also, facial dysmorphism, milestone regression and under-nutrition were obvious. Ascites and hepatosleenomegaly were added characteristic features. Patient weighed $8 \mathrm{kgs}$. Liver was scaled to measure $8 \mathrm{~cm}$ while spleen was $10 \mathrm{~cm}$.On ophthalmic examination, pupil showed sluggish reaction. No response were seen with respect to the stimuli, and the reflexes were quite poor.

\section{Laboratory investigations:}

\begin{tabular}{|l|l|}
\hline Haemoglobin & $10.78 \mathrm{~g} \%$ \\
\hline Total leukocyte count & $17,510 / \mathrm{mm}^{3}$ \\
\hline Platelets & 81,000 \\
\hline Bilirubin - Total & $9.9 \mathrm{mg} \%$ \\
\hline Bilirubin - Direct & $5.1 \mathrm{mg} \%$ \\
\hline Bilirubin -Indirect & $4.8 \mathrm{mg} \%$ \\
\hline SGOT & $105 \mathrm{IU} / \mathrm{L}$ \\
\hline SGPT & $36 \mathrm{IU} / \mathrm{L}$ \\
\hline Alkaline Phosphatase & $232 \mathrm{IU} / \mathrm{L}$ \\
\hline
\end{tabular}


Hypoalbuminemia was also quite evident, with albumin value as low as $1.9 \mathrm{~g} / \mathrm{L}$. Abdominal USS was done. The reports revealed hepatospleenomegaly with large mesenteric lymphnodal masses, retroperitoneal lymphnodes, enlarged porta peripancreatic lymphnodes with moderate ascites.

Diagnostic and Therapeutic Concerns: These investigations in totality can be cumulated to diagnose this child as a Niemann pick disease- Type A. Although genetic testing, bone marrow analysis \& sphingomyelinase assay would have served as assets in its diagnosis.Patient was closely monitored and symptomatic treatment was provided. But unfortunately the child passed away, and it's said to be due to hepatic failure.

\section{Discussion}

The onset of Niemann pick disease type A occurs at a very young age, developing symptoms as early as 3 months. This progressive neurodegenerative disease rapidly conquers the individual with massive hepatospleenomegaly, interstitial lung disease, cherry red macula and eventually death within the age of 3 years, making it a fatal disorder of infancy. Cases of type A are usually rare, with type B predominating and sometimes an intermittent type involving A \& B in the same individual. Although type A and type B are caused by the same enzymatic deficiency, the 2 forms are completely opposite ends of a contium. Niemann pick disease type B often presents in mid-childhood. The signs and symptoms are most probably same as type A, but unlike type A the severity is minimal. Additionally, they manifest thrombocytopenia, short stature and delayed bone age. The survival rate in type $\mathrm{B}$ is usually up to late adulthood, yet the fear of death is always a threat in these individuals. Apparently, Niemann pick disease type $\mathrm{C}$ is thought to be manifested in the early adulthood. Despite the fact, there is a variation in their genetic aetiology, the clinical presentation of Niemann pick disease type C, are similar to type A \& B. Furthermore, they also characterize ataxia, vertical supranuclear gaze palsy and dystonia. ${ }^{[3]}$ Considering the fact, Niemann pick disease is a genetic disorder, its management is quite often a challenging task. As for type A, it holds no options for therapy. Symptomatic treatment is mainstay, yet fatality cannot be escaped. Niemann pick disease type B on the other hand are tackled with Statins to control cholesterol levels, blood transfusion are helpful in thrombocytopenia and supplemental $\mathrm{O}_{2}$ for interstitial lung disease. ${ }^{[3-4]}$ Likewise, type $\mathrm{C}$ can be looked after with the same. Moreover, a new drug has been approved for type $\mathrm{C}$, MIGLUSTAT, although not by FDA ${ }^{[5]}$ It has shown to withhold the neurological symptoms of type C.

\section{Conclusion}

NPD type $A$ is a fatal disease of infancy. No treatment is till date available for it. Early diagnosis and management of complications usually plays a significant role to add extra years to their life.

\section{References}

[1]. $\quad$ Marie T Vanier, Orphanet J Rare Dis, 2010, 16 (5), 1750-1172

[2]. William L. Nyhan, Bruce A. Barshop, and Pinar T. Ozand, Atlas of Metabolic Diseases, 2005, 2, 635-650

[3]. Shubhankar M, Sunil K. A, Bikash R.P. and Shantanu K.M. et al, Scholars Academic Journal of Biosciences, 2014, 2(10): 728-730

[4]. E. H. Schuchman, Journal of Inherited Metabolic Disease, 2007, 30, 654

[5]. MC Patterson, The Lancet, Neurology, 2007,6 (9), 765-772 